\title{
Contrôle des débits dans les réseaux d'adduction d'eau
}

A la suite d'une expertise sur les débits d'eau potable à Paris, confiée par la SAGEP, la C.E.P. et la S.P.E. à Monsieur G. MaURIN, Président de la Société Hydrotechnique de France, ce dernier a suggéré de consacrer une ou deux journées d'études à ces problèmes.

La Société hydrotechnique de France (S.H.F.) et l'Association Générale des Hygiénistes et Techniciens Municipaux (A.G.H.T.M.) ont organisé un colloque à l'Ecole des Ingénieurs Hydrauliciens et Mécaniciens de Grenoble. les 20 et 21 avril 1994.

La première journée, qui réunissait 120 participants, a été consacrée au contrôle des débits dans les réseaux urbains d'adduction d'eau, aux résultats obtenus lors de l'expertise parisienne, mais aussi aux connaissances récentes acquises par des constructeurs de débitmètres, des laboratoires d'études, et par des exploitants de réseaux.

Les exposés ont concerné surtout les mesures par débitmètres à ultra-sons, mais aussi les compteurs d'eau, les débitmètres électro-magnétiques, la normalisation des termes employés dans ce domaine, et les possibilités d'utilisation rationnelle des télémesures dans la gestion d'un réseau d'eau potable.

La première remarque qui s'impose à l'examen des communications et à la suite des discussions en séance est la diversité des précisions annoncées pour les mesures :

- mieux que $1 \%$ pour les fabricants d'appareillage ;

- $1 \%$ à $5 \%$ pour les laboratoires ;

- $2 \%$ à $10 \%$, et même $20 \%$ à $30 \%$ dans certains cas de vieux réseaux, pour les exploitants, dans les conditions industrielles d'installation.

\section{Compteurs d'eau}

Dans la première communication, Monsieur A. Costes (SAGEP) décrit les différents types de compteurs : volumétriques, à turbines, Woltman, combinés ou proportionnels, et analyse, de faşon sommaire car ce n'était pas le sujet principal du colloque, leurs avantages et inconvénients, leur précision et les méthodes d'étalonnage.

\section{Débitmètres à ultra-sons}

Le principe de ces appareils (mesure de la différence de temps mis par une onde ultrasonore à $45^{\circ}$, dans le sens du courant et en sens contraire), les formules utilisées et les causes d'erreur apparaissent dans plusieurs communications ( 1 - Costes, 2 - MULTON, 4 - BIAGGI el DELATTRE, 5 - JOSSINET, 7 - PERILLAT et HERIGAULT, présentée par M. ROBERT, 8 - GiRAUd, HEBER-SUFFrin, et GUILlERMOU). II y a unanimité pour souligner que les appareils multicordes ont de meilleures performances que les débitmètres monocordes, surtout quand on ne dispose pas de conditions hydrauliques amont et aval satisfaisantes ; Monsieur
JOSSINET cite les ordres de grandeur des précisions obtenues en laboratoire, dans des conditions identiques, pour un débit égal à $10 \%$ du débit nominal des appareils :

- $3 \%$ à $5 \%$ pour un monocorde :

$-1,5 \%$ pour un tricorde

- 0,5\% pour 4 cordes.

Les exploitants de réseau mettent cependant en valeur les avantages des débitmètres monocordes, surtout quand on dispose de grands alignements sans obstacles qui permettent l'obtention d'une précision suffisante : installation possible sur des conduites en charge sans arrêt de l'installation, prix d'achat et frais de mise en service très faibles, entretien simple et économique.

\subsection{Electronique}

Les appareils analogiques semblent condamnés, au profit des débitmètres numériques : on a en effet constaté d'importantes dérives dans le temps liées en partie aux contacts des potentiomètres. Pour les appareils numériques, même si on n'atteint pas toujours la précision de I nanoseconde annoncée par les constructeurs, et si on a constaté une dispersion de 20 nanosecondes entre plusieurs mesures de trajet en eau immobile (7 - PERILLAT-HERIGAULT), la précision globale peut être meilleure que $1 \%$, du fait que la mesure du temps est réitérée plusieurs fois pour une même mesure.

Il est utile de prévoir un filtrage de l'alimentation électrique, et une protection électro-magnétique (7 - HERIGAULT et 8 - GiRAUD, HEBER-SUFFrin, GUILLERMOU).

\subsection{Géométrie}

La très bonne connaissance de tous les paramètres géométriques qui interviennent dans un débitmètre à ultrasons est fondamentale : distance l entre sondes, diamètre réel $D$ de la conduite dans la section de mesure, distance $d$ entre plans de sondes, les derniers paramètres intervenant par leurs carrés.

Un calcul théorique (5 - JOSSINET) montre qu'on peut évaluer l'erreur de justesse liée aux paramètres géométriques à moins de $2 \%$ pour les débitmètres à sondes accrochables extérieurement, et à moins de $0,8 \%$ pour des sondes intrusives.

En fait, dans le cas de sondes intrusives installées sur des conduites en charge en adoptant sans vérification le diamètre nominal de la conduite et les valeurs théoriques calculées pour l et d, les erreurs constatées réellement ont parfois dépassé $10 \%$ (com. $n^{\circ} 7$ et 8$)$.

Si on veut obtenir une bonne précision, il est donc indispensable d'installer les sondes sur une conduite vidangée, de faire les vérifications et mesures avec minutie depuis l' intérieur de la conduite (com. 7 et 8), puis de fixer de manière indéréglable les sondes en enfoncement et en rotation. 
Certains constructeurs orientent leurs sondes à l'aide d'un rayon laser.
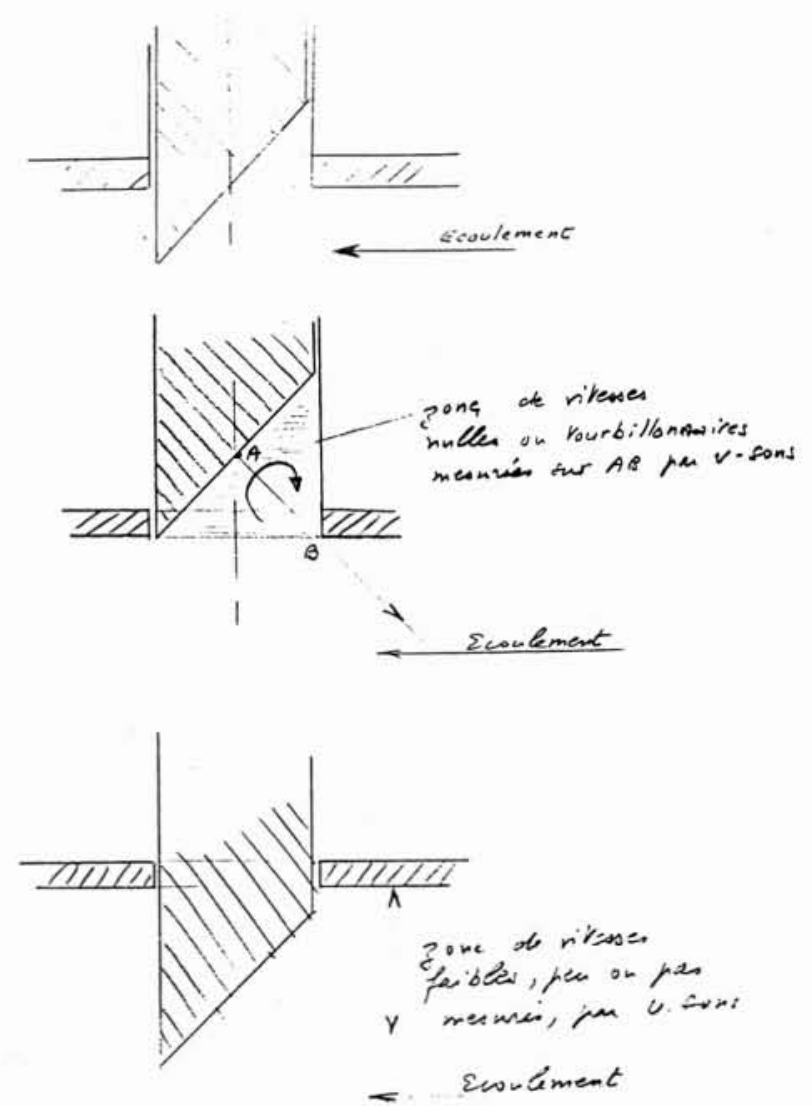

1. Influence d'un mauvais positionnement des sondes.

\subsection{Enfoncement des sondes (voir fig. 1)}

Le respect du positionnement des sondes suivant les indications du constructeur est essentiel ; en effet, aussi bien la théorie que les expériences faites in situ et au laboratoire, montrent que la mesure par ultra-sons de la vitesse moyenne est entâchée d' une erreur dont l'ordre de grandeur est dlll, si dl est l'écart d'enfoncement par rapport à la préconisation du fabricant, en surestimation si la sonde est trop avancée dans l'écoulement, en sousestimation si elle est en retrait dans le métal (com. 2,7 et 8). On ne connaît pas avec précision l' influence des tourbillons qui se forment autour de la tête de sonde.

\subsection{Conditions hydrauliques}

Les conditions d'installation hydraulique ont une grande influence sur la précision des mesures, surtout pour les débitmètres monocordes, et c'est surtout dans ce domaine qu'il faudra améliorer les connaissances.

COEFFICIENT $\mathrm{kH}$ : Le débitmètre à ultra-sons mesure la vitesse moyenne sur la corde de mesure, et non la vitesse "débitante " $Q / S(Q$ débit, $S$ section de mesure). Si on se contente de faire le produit de la vitesse mesurée par la section de mesure, on surestime le débit car on attribue le même poids à la vitesse maximale au centre qui ne concerne en fait qu' une très petite section, et aux vitesses faibles près des parois, qui s'appliquent à de grandes sections. Il est donc nécessaire d'introduire un coefficient de correction $\mathrm{kH}$, qui varie de 1,04 à 1,08 suivant le nombre de REYNOLDS et la rugosité de la paroi (com. $n^{o s} 2,5,7$ et 8 ).

L'expérience acquise à Paris, grâce à l'exploration du champ des vitesses à l'aide de 13 moulinets, confirme bien les ordres de grandeur des coefficients généralement adoptés ( $k H$ variant de 1,05 à 1,07 pour un nombre de REYNOLDS évoluant de 1000000 à 100000 dans d'excellentes conditions hydrauliques, alignements droits de plus de 100 D) (fig. 2).

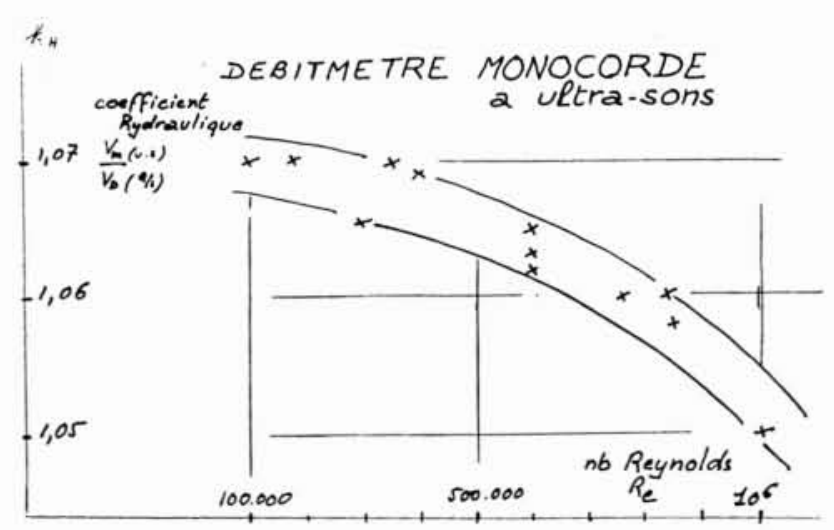

2. Influence des conditions hydrauliques pour les débitmètres monocordes à ultra-sons.

On n'a pas pu mettre en évidence l'influence de la rugosité, qui paraît faible.

INFLUENCES HYDRAULIQUES: L'influence des perturbations amont et aval est encore insuffisamment connue, en particulier leur évolution avec le débit, pour un type de perturbation donné. On a constaté, lors d'une exploration du champ des vitesses par moulinets, lors de la vérification d'un débitmètre monocorde à ultra-sons d'un des ponts de Paris, installé dans des conditions difficiles (environ à $10 \mathrm{D}$ de 2 coudes, un à l'amont, l'autre à l'aval) que la vitesse maximale se situait sur la rive droite pour les forts débits, sur la rive gauche pour des débits plus faibles, alors qu'il y avait une relative symétrie de vitesses pour des débits moyens; le coefficient $k H$, dans la section de mesure au moulinet, aurait varié de 1,00 à 1,025 (com. $n^{\text {os }} 7$ et 8).

Toute une série d'essais, avec des débitmètres de marques différentes, a été menée en laboratoire par E.D.F., en installant des obstacles variés à l'amont et à l'aval de débitmètres à ultra-sons (coudes dans des plans différents, convergents, divergents...). Les résultats détaillés figurent dans la communication $n^{\circ} 5$ de Monsieur JOSSINET ; on notera en particulier une erreur de $30 \%$ induite par un divergent, et ceci n'a rien d'étonnant; on a représenté sur la figure 3 un cas purement théorique: dans une conduite de $0,5 \mathrm{~m}^{2}$ de section, où passe un débit de $0,5 \mathrm{~m}^{3} / \mathrm{s}$, on introduit successivement un diaphragme de $0,5 \mathrm{~m}^{2}$, puis un disque central de $0,5 \mathrm{~m}^{2}$; on suppose que les vitesses sont uniformes et égales à $1 \mathrm{~m} / \mathrm{s}$ dans les 
écoulements, nulles à l'aval des obstacles, ce qui n'est pas exact, mais introduit par rapport à la réalité des erreurs inférieures à $10 \%$. Le calcul montre que les vitesses moyennes qui seraient mesurées par des débitmètres monocordes à ultra-sons placés immédiatement à l'aval des obstacles seraient de $0,80 \mathrm{~m} / \mathrm{s}$ pour le diaphragme et de $0,29 \mathrm{~m} / \mathrm{s}$ pour le disque central, soit des écarts de $40 \%$ par rapport à la vitesse débitante de $0,5 \mathrm{~m} / \mathrm{s}$ ( $\mathrm{kH}$ de 1,42 et 0,58$)$.

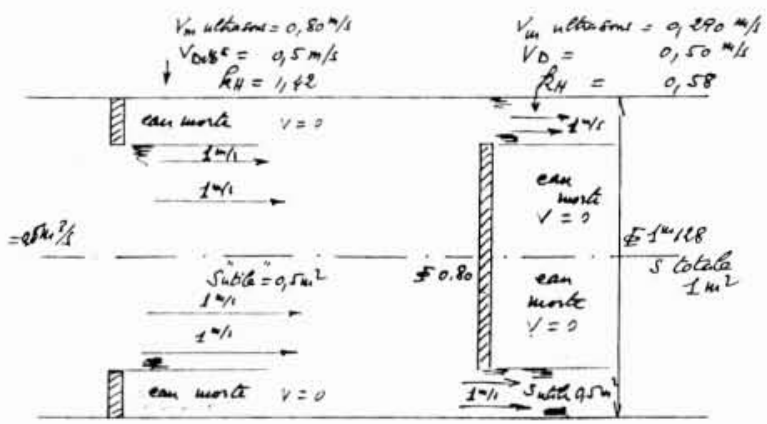

3. Exemple de situation où les conditions hydrauliques créent des écarts de mesure importants.

J. BIAGGI et S. DELATTRE font état dans la communication $n^{\circ} 4$ d'erreurs importantes, pas toujours explicables rationnellement, dans le cas de vieux réseaux en exploitation.

M. Jossinet a étudié la possibilité d' améliorer les résultats à l'aval d'une singularité, en explorant à l'aide de débitmètres à sondes accrochables les résultats obtenus sur différentes cordes d'une même section, avant d'installer les sondes intrusives définitives.

Tous les participants reconnaissent les améliorations apportées dans des cas hydrauliques difficiles par l'utilisation de débitmètres multi-cordes ; malheureusement, ces appareils sont plus onéreux et d'installation plus délicate.

\section{Débitmètres électromagnétiques}

Le principe de mesure (modification par l'écoulement du champ magnétique créé par 2 électrodes diamétrales dans la section de mesure), et la description de ces appareils apparaissent dans les communications $n^{\circ} 1$ (COSTES) et $n^{\circ} 3$ (BAUDET).

L'opinion générale est que ce type d'appareil est beaucoup moins sensible aux singularités hydrauliques que les débitmètres monocordes à ultra-sons; en effet les lignes de champ créées dans l'écoulement, même si elles sont induites par deux électrodes diamétrales, concernent toute la section de mesure et non une seule corde.

En outre, ces appareils sont souvent montés entre deux manchettes coniques de raccordement, ce qui améliore les conditions d'écoulement (et réduit les prix); le tarage en laboratoire est effectué avec ces manchettes, la précision étant meilleure que $1 \%$.

Les bons résultats obtenus actuellement avec ce type d'appareillage sont dus essentiellement aux progrès de l'électronique, qui permettent la mesure précise de microvolts.
Toutefois, lors de l'expertise parisienne, on a constaté des erreurs de plus de $3 \%$ à l'occasion du retarage en laboratoire d' un débitmètre électromagnétique analogique d'un modèle ancien et des écarts inadmissibles par rapport au tarage du constructeur sur un appareil neuf, lors d' une vérification en laboratoire.

Ce type de débitmètre est plus cher qu'un appareil monocorde à ultra-sons et son coût d'installation important, car il est indispensable de prévoir sa démontabilité pour des vérifications ultérieures en laboratoire; un montant d'installation de 800000 francs a été indiqué dans un cas difficile de Paris.

\section{Contrôles en exploitation}

Pour tous les types de débitmètres, l'importance de certaines erreurs décelées lors des expertises récentes montre $q u ' i l$ est indispensable de procéder à des vérifications périodiques, in situ ou en laboratoire; on trouvera dans les communications 7 et 8 des informations détaillées sur les mesures possibles de comparaison (moulinets, méthode thermodynamique, vidange de rédervoirs...), et dans la communication $n^{\circ} 10$ de Monsieur GUILLERMOU les possibilités modernes de contrôle par utilisation des télémesures.

La détection de la dérive du "zéro " des appareils reste la plus difficile à mettre en évidence, car il est parfois impossible d'affirmer que le débit est bien nul dans la conduite après fermeture des vannes; un tel écart est en général constant en valeur absolue quel que soit le débit dans la conduite.

On doit signaler que des écarts de $2 \%$ ont été trouvés entre indicateurs et totalisateurs d'un même appareil pour une même mesure.

\section{Utilité des contrôles et mesures}

L'amélioration de la précision des mesures est évidemment indispensable en raison de l'importance des montants facturés (sur l'ensemble de Paris une erreur de $1 \%$ représente 3 millions de $\mathrm{m}^{3}$ d'eau par an, soit près de 30 millions de francs), mais elle permet aussi l'amélioration de la gestion, la détection et la suppression des fuites.

Dans le cas très favorable du réseau d'eau potable de Paris, où la plupart des canalisations sont visitables par les égouts, l'amélioration des contrôles a permis, en quelques années, une diminution de consommation annuelle de 35 millions de $m^{3}$ d'eau (com. $n^{\circ} 8$ ).

\section{Normalisation}

La communication $n^{\circ} 9$ de J.L. DEMASSUE fait le point sur les travaux d'un groupe de travail de l'A.G.H.T.M. qui s'est attaché à "normaliser "la définition et l'utilisation des paramètres utilisés pour la bonne gestion d'un réseau. Elle met en évidence la nécessité, mais aussi la difficulté de parler le même langage : rendements, performances, pertes, fuites, identification des volumes perdus, détournés, gaspillés...

Si la valeur absolue des différents ratios de gestion donne une photographie de l'état d'un réseau, leur évo- 
lution dans le temps permet de mieux cerner son vieillissement et l' intérêt de travaux éventuels. Ces études permettent aussi de donner les ordres de grandeur de certains paramètres, tels que les limites des pertes linéaires admissibles pour divers types de réseaux, les valeurs relatives des fuites des branchements dans le bilan total...

\section{Télésurveillance et exploitation des télémesures}

Monsieur J.L. GULLERMOU, dans la communication $n^{\circ} 10$, fait le bilan de l'utilisation en temps réel des télémesures précises dans la surveillance et l'optimisation du réseau de Paris.

La détection des anomalies utilise plusieurs moyens en parallèle : établissement de bilans journaliers, hebdomadaires ou mensuels, contrôle de cohérences à partir de sommes algébriques des indications de différents comptages. Un exemple d'utilisation d'un programme à base $d$ 'intelligence artificielle pour comparer prévisions et réalisations ouvre d'intéressantes perspectives.

De même la comparaison d'un petit réseau témoin "VERCINGÉTORIX " en excellent état, bien connu et surveillé, avec le reste du réseau ouvre la voie à des possibilités nouvelles, en donnant aussi l'ordre de grandeur de ce qui pourrait être considéré comme le rendement d'un réseau idéal : $97 \%$ ?

\section{Perspectives d'avenir}

La plupart des exploitants ont souligné la nécessité, pas toujours admise actuellement par les bureaux d'étude. d'inclure l'analyse du contrôle des débits et la définition des moyens de mesures dans la conception des réseaux dès l'origine : alignements droits sans obstacle, regards de visite, démontabilité...
En ce qui concerne les débitmètres à ultra-sons, il est urgent de faire les études et mesures nécessaires en laboratoire pour définir avec précision les conditions hydrauliques indispensables pour l'obtention d'une bonne précision, et ceci sur toute la gamme des débits à mesurer; il serait utile de donner des indications sur la meilleure position des sondes en fonction de diverses singularités hydrauliques.

Il serait intéressant d'étudier un dispositif permettant de ne pas perturber l'écoulement à l'extrémité des sondes, par exemple en plaçant les sondes en retrait dans le métal de la conduite, et en colmatant le vide avec un matériau de densité 1 perméable aux ultra-sons, pour reconstituer la paroi de la conduite.

Les études sur la précision des débitmètres électromagnétiques, en particulier en présence d'obstacles proches dans l'écoulement, et ceci pour toute la gamme de débits, ne semblent pas avoir été suffisamment avancées; la position optimale des électrodes en fonction des singularités hydrauliques serait à définir. Un des participants a souhaité que soient étudiées la réponse et la précision des différents débitmètres en présence de débits pulsés.

\section{Conclusion}

Les débitmètres électro-magnétiques, les débitmètres à ultra-sons, même monocordes, installés dans les conditions requises, ayant fait l'objet des contrôles géométriques nécessaires et des tarages préconisés, sont capables d'assurer la mesure et le contrôle des débits dans des conditions industrielles avec une bonne précision, meilleure que $2 \%$, dans la mesure où ils font l'objet d' une maintenance et de contrôles programmés.

H. ANDRE

\section{Discussions de la $\mathbf{1}^{\text {ère }}$ journée}

\section{Discussion des communications $1,2,3,4$ et 5 Secrétaire de séance : M. ROBERT}

Sont intervenus: Messieurs ANDrÉ, BaUdet, BIAGgI, Bonazzi, Bouis, Cazenave, Chaigne, Costes, Delhaye, Demassue, Fritsch, Gasc, Jossinet, Maurin, Multon, Poisson, Schulhof, Tiret, Tournier, Willemetz.

\section{Procédé sing-around}

Dans les formules de mesures de vitesse par ultra-sons, pour éliminer la célérité du son, qui varie avec la température, on est amené à faire intervenir, non pas une différence de temps, mais des différences de fréquences $1 / t$, ou le paramètre $\mathrm{dt} / \mathrm{t} 2$ ( $\mathrm{dt}$ différence des temps de parcours, $\mathrm{t}$ temps moyen de trajet).
Certains constructeurs ont adopté le procédé «singaround " qui fait intervenir directement $1 / \mathrm{t} 1$ - 1/t2 ; l'inconvénient est qu'on perd tout le train d'ondes quand le signal rencontre une perturbation telle que bulle ou impureté ; en général ces particules ne sont pas trop gênantes dans les mesures classiques par ultra-sons, on arrive même à faire des contrôles dans les égouts ! Les micro-bulles sont plus gênantes.

\section{Résistivité}

Avec les débitmètres électro-mangétiques, on peut, moyennant quelques précautions, détecter 1 microsiemens ; il n'y a donc aucun problème pour la mesure de débits d'eau, mais cette technologie n'est pas utilisable avec des produits pétroliers non conducteurs. 


\section{Ultra-sons, paramétrage du coefficient hydraulique $\mathrm{kH}$}

Les débitmètres modernes, équipés de microprocesseurs, permettraient à terme le calcul de $\mathrm{kH}$, si on les renseigne sur certains paramètres tels que la rugosité, mais on envisage toujours la possibilité d'introduire manuellement les valeurs de $\mathrm{kH}$; on pourrait prochainement introduire la courbe d'évolution de $\mathrm{kH}$ en fonction du débit dans le débitmètre.

\section{Normalisation}

Tous les participants souhaitent l'édition prochaine d'une norme précisant en particulier les conditions précises d'installation des débitmètres, les valeurs des alignements droits à respecter à l'amont et à l'aval ; on regrette la très faible participation française dans les groupes de normalisation. On espère qu'une norme ISO sera éditée en 1995, mais il reste beaucoup d'études à faire, surtout pour les appareils mono-cordes, pour bien connaître l'influence, sur toute la gamme des débits, d'éléments perturbateurs variés.

\section{Précisions}

Les mesures sont d'autant plus précises que les diamètres des conduites sont plus gros, et que les vitesses sont plus élevées ; c'est en effet le nombre de nanosecondes correspondant à la différence des temps de trajets des ondes ultra-sonores qui permet le calcul de l'erreur.

Pour les débitmètres électromagnétiques, des abaques concernant la précision sont généralement fournies avec l'étalonnage.

\section{Réétalonnages}

Pour les constructeurs, il faut essentiellement surveiller l'évolution de l'électronique ; pour les exploitants il paraît indispensable de réétalonner périodiquement tout l'appareillage, soit en laboratoire après démontage, soit par une méthode de contrôle in situ.

\section{Mesure de débits pulsés}

Dans le cas des appareils à ultra-sons, pour une cadence d'émission de $256 \mathrm{~Hz}$, le temps de réponse de l'électronique est de l'ordre de 0,1 seconde, de sorte que si la pulsation du débit n'est pas trop rapide, on devrait faire des mesures correctes tant que le régime reste turbulent.

\section{Protection des appareils}

Il faut étudier les moyens de rendre les réglages des appareils inaltérables par inadvertance... ou par malveillance (réglage des paramètres, positionnement des sondes...).

\section{Etablissement de profils de vitesses par effet Doppler}

Des contrôles des profils de vitesse sont possibles par effet « DOPPLER " de manière non intrusive, ce qui présente un gros intérêt, mais le manque de particules en suspension dans l'eau potable semble apporter parfois certaines difficultés.

\section{Electronique. Environnement électrique}

$\mathrm{Si}$, pour la partie hydraulique on cite des erreurs possibles supérieures à $10 \%$, alors qu'en électronique on raisonne en erreurs de millièmes, il n'est cependant pas inintéressant de mieux connaître et de visualiser les signaux incidents, d'utiliser éventuellement des principes d'intercorrélation. Il faut tenir compte du milieu environnant ; les participants ont eu des résultats très variables : succès lors de mesures par U.S. sur un alternateur (100 MVA, 4000 A), ennuis à proximité de pompes.

\section{Discussion des communications 6,7 et 8 Secrétaire de séance : Monsieur FRITSCH}

Sont intervenus : Messieurs : Barbier, CAZEnave, Costes, Fruchart, Guillermou, Maurin, Multon, Robert, VALIBOUSE

\section{Contrôles par moulinets}

La mesure du débit avec des moulinets est-elle assez précise pour servir de référence dans le contrôle de débitmètres à ultra-sons ou électromagnétiques, et en particulier la couche limite n'a-t-elle pas d'influence sur les moulinets les plus proches de la paroi ?

Les normes d'utilisation des moulinets, qui ont été établies en tenant compte de l'expérience de milliers de mesures, en particulier pour le contrôle des rendements des turbines conduisent à mettre les moulinets hors de la couche limite, et concluent à une précision meilleure que $2 \%$.

\section{Amélioration de la gestion des réseaux}

Dans le cas de Paris, la gestion par plusieurs partenaires, la nécessité de bien connaître les volumes échangés et une campagne de mesures très poussées dans le cadre d'une récente expertise ; ont permis de faire passer le rendement des réseaux de $78 \%$ en 1985 à $90 \%$ en 1994, après un très gros effort de recherche et de limitation des fuites.

\section{Précision de l'électronique U.S.}

On peut s'étonner de la dispersion de 20 nanosecondes avancée lors des contrôles d'expertise alors que la précision attendue sur la mesure de la différence de temps de trajet est de l'ordre de une nanoseconde; ces résultats ont été obtenus en comparant le simulateur de sondes du constructeur et des compteurs haute résolution (RACAL 1992 et PHILIPS 6671) testés en parallèle sur un générateur RHODE et SCHWARTZ, autorisant une dispersion de 0,1 nanosecondes pour une période de 100 microsecondes; ces résultats ont été confirmés dans de l'eau immobile à $23^{\circ}$.

Ces divergences, qui ne remettent pas en cause une précision meilleure que $1 \%$ pour l'ensemble de l'appareil 
U.S., peuvent avoir plusieurs causes : fonction de transfert du transducteur et son câble de liaison (un signal électrique parcourt $20 \mathrm{~cm}$ en une nanoseconde dans un câble coaxial), certains composants travaillent à la limite, réponses différentes des 2 sondes amont et aval...

\section{Sondes U.S. installées en atelier sur des manchettes}

Pourquoi ne pas avoir installé en 1985 sur le réseau parisien des manchettes prééquipées de sondes U.S. en atelier, ce qui aurait évité des erreurs géométriques et les problèmes d'enfoncement?

Il fallait alors installer très rapidement et à des coûts raisonnables plusieurs dizaines d'appareils sans interruption du réseau; les connaissances en la matière étaient celles des études présentées en 1980 au congrès A.I.D.E. (on avançait alors comme suffisants des alignements droits de 3D et 2D !), on ne connaissait pas l'influence de l'enfoncement des sondes. On a installé des sondes intrusives sur le réseau en charge sans aucune interruption.

\section{Régularisation de l'écoulement}

On peut envisager la mise en place de guide-eaux en amont des débitmètres, pour éviter de longs alignements droits, mais les exploitants préferrent utiliser dans les cas limites des débitmètres multi-cordes (1), plutôt que d'introduire des obstacles et des pertes de charge dans l'écoulement.

\section{Discussion des communications 9 et 10 Secrétaire de séance : Monsieur FRITSCH}

Sont intervenus : Messieurs : BARBIER, BIAGgi, Demassue, GUILlermou et ROBERT.

Le réseau de référence VERCINGÉTORIX a été choisi pour sa petite taille, sa proximité des bureaux de la S.P.E., sa représentativité en densité de compteurs et son parfait état après travaux.

Le système d'analyse «DETECTOR » ne prend en compte pour les prévisions de consommation ni la température, ni les prévisions météorologiques, car à Paris la météo a relativement peu d'influence sur la consommation (peu d'arrosage de jardins). L'utilisation, pour les prévisions, de la consommation de la veille donne satisfaction.

\section{Pertes}

Sauf dans le cas particulier de Paris, les branchements sont responsables d'une grande partie des pertes volumiques: fuites faibles, mais permanentes et difficiles à détecter.

(') ou électromagnétiques. 